\title{
"Régime international, déforestation évitée et évolution des politiques publiques et privées affectant les forêts dans les pays du Sud »
}

\section{Compte rendu d'atelier (Paris, 21-23 novembre 2007)}

\author{
Alain Karsenty \\ Économiste, CIRAD, Ressources forestières et politiques publiques, 34398 Montpellier cedex 5, France
}

Cet atelier international ${ }^{1}$ a réuni 140 participants venant du monde entier quelques jours avant la $13^{\mathrm{e}}$ Conférence des Parties (CoP 13) de la Convention-cadre des Nations unies sur les changements climatiques, qui s'est déroulée à Bali en décembre 2007.

La raison d'être de cet atelier découlait d'un ensemble de constats. Les politiques forestières mises en œuvre dans les pays tropicaux disposant de vastes surfaces boisées semblent converger dans leurs objectifs et dans les modalités de gestion. Cette convergence ferait suite à l'utilisation d'un certain nombre d'instruments : la concession forestière pour l'organisation de la production dans le cadre d'objectifs affichés de gestion durable de la ressource; des dispositifs normatifs privés transnationaux, comme la certification forestière, qui s'impose progressivement par le biais des marchés; la perspective d'un mécanisme de la déforestation évitée, portée par des négociations qui ont été ouvertes à l'occasion de la Convention-cadre des Nations unies sur les changements climatiques. Couplé avec l'existence d'institutions (Forum des Nations unies sur les forêts [FNUF], Organisation internationale des bois tropicaux [OIBT]...) et d'accords internationaux

\footnotetext{
Auteur correspondant : alain.karsenty@cirad.fr

${ }^{1}$ Organisé conjointement par le Centre de coopération internationale en recherche agronomique (Cirad), l'Institut du développement durable et des relations internationales (IDDRI), le Center of International Forestry Research (CIFOR) et le groupement d'intérêt public Écosystèmes forestiers (GIP ECOFOR). Un compte rendu collectif de cet atelier (dont une version anglaise a été publiée en 2008 dans International Forestry Review, 10, 3, 424-428) est consultable sur les sites Internet du Cirad http://www.cirad.fr/ur/ressources_forestieres (Publications et colloques - Ateliers) et de l'IDDRI http://www.iddri.org (Publications, Collections, Idées pour le débat, 2008, nº 7).
}

(Accord international sur les bois tropicaux [AIBT]...), l'ensemble de ces éléments peut s'analyser sous l'angle de l'émergence ou de la consolidation d'un régime international des forêts, dont il convient d'analyser l'efficacité au moment où le rôle des forêts dans la problématique des changements environnementaux globaux est souligné avec force.

\section{Les forêts des pays du Sud : des questions multiples}

Les principales questions auxquelles ont tenté de répondre les participants étaient nombreuses :

- Quels sont les indicateurs qui permettent d'identifier la constitution d'un régime international des forêts ${ }^{2}$ ?

- Dans quelle mesure les éventuels régimes internationaux en voie d'émergence dans le domaine de l'environnement influent-ils sur la construction des

\footnotetext{
${ }^{2}$ La définition la plus connue de régime international a été formulée en 1983 par Stephen Krasner : «Un régime international est un ensemble de normes, règles, principes et procédures de décision qui forme les anticipations des agents et guide leurs actions. » Notons également la proposition de Marie-Claude Smouts lors de son intervention pendant l'atelier : «Un régime se définit par des normes de comportement : il existe lorsque les acteurs ne peuvent plus prendre leurs décisions de manière totalement autonome. Cela ne veut pas dire que le comportement des acteurs sera conforme à celui qui est inscrit dans le régime, mais qu'il existe une sorte de loi surplombante par rapport à laquelle ils sont amenés à se définir et qu'éventuellement, ils peuvent avoir à payer un certain prix s'ils s'en écartent ouvertement. En bref, on n'est plus totalement libre de faire n'importe quoi ; la transgression peut avoir un coût. »
} 
politiques publiques en matière de forêt et d'environnement des grands pays forestiers en développement (Brésil, Bolivie, pays d'Afrique centrale, Indonésie, Malaisie...)?

- Y a-t-il une convergence des politiques publiques des grands pays forestiers du Sud? Si oui, sur quels points? Quels sont la place et l'impact des dispositifs privés (comme la certification ou les engagements volontaires) dans les évolutions en cours et comment doit-on les interpréter?

- Quels sont les éléments de divergence des politiques nationales et les raisons des différences de situation constatées? Comment les représentations collectives nationales influent-elles sur ces choix?

- Est-ce que la place grandissante que prennent les forêts dans le débat international sur l'environnement, et en particulier à travers les projets de versements financiers aux pays en fonction de la réduction de la déforestation ("déforestation évitée »), va accélérer la constitution d'un régime international des forêts tropicales? Quelles sont les marges de manœuvre réelles, notamment en termes politiques et sociaux, des gouvernements en matière de modification des politiques publiques pour infléchir le taux de déforestation? Comment évaluer les coûts d'une réduction de la déforestation obtenue par le biais de «paiements pour services environnementaux »?

Des interventions et des débats, il est possible d'extraire un certain nombre de points forts en référence à ces questions.

\section{L'émergence d'un régime international des forêts}

La notion de régime international des forêts est bien floue. S'il en existe incontestablement des éléments, ils sont empruntés à d'autres régimes (celui de la biodiversité, celui de l'aménagement, celui dit climat-forêts, celui des peuples autochtones). De ce fait, ils sont dispersés, hétérogènes. De plus, il n'y a pas de hiérarchie entre eux et les protagonistes de chacun d'eux se préoccupent assez peu de ce qui se passe dans les domaines connexes. Or, les activités des mêmes acteurs se déroulent dans des régimes multiples, avec des intérêts parfois très contradictoires. La notion de régime ne permet pas de rendre compte de la complexité de ces interdépendances.

Pour juger de l'efficacité d'un éventuel régime international des forêts, il faudrait des critères partagés d'évaluation. Or, il y a des désaccords sur la hiérarchie de ces critères, notamment sur les questions de l'exploitation industrielle du bois, de l'aménagement forestier, des droits des populations ou de la lutte contre la pauvreté. En tout état de cause, le régime international «en pointillés », que l'on peut discerner en analysant les différents domaines, n'est pas très efficace en ce qui concerne un certain nombre de grandes questions, comme la déforestation.

Les politiques nationales de nombreux pays forestiers présentent des éléments de convergence à travers l'adoption d'instruments, comme les concessions forestières, l'aménagement forestier, les instruments de vérification de la légalité, la décentralisation ou la foresterie communautaire. Un facteur important de convergence autour de la notion de gestion durable est apporté par les dispositifs internationaux privés, comme la certification forestière. Le poids du Forest Stewardship Council (FSC), en particulier, a été souligné ; son rôle pour la constitution d'un référentiel global de la gestion durable est incontestable, malgré la relative modestie des surfaces de forêts naturelles tropicales aujourd'hui certifiées.

\section{De fortes spécificités locales}

Mais la convergence ne s'étend pas au-delà de certains segments, car les politiques publiques restent profondément marquées par des choix collectifs nationaux et des manières locales de « faire de la politique ».

- En Asie du Sud-Est, les options concernant la forêt restent soumises à la demande industrielle, aujourd'hui tournée vers le développement de la pâte à papier et vers les cultures industrielles de rente. En Indonésie, la décentralisation de la gestion des forêts a entraîné une grande confusion, sans qu'un système local viable n'émerge sur les décombres de l'ancien, qui, centré sur l'exploitation industrielle des forêts, avait conduit le pays à devenir le champion mondial du contreplaqué, avant le quasi-effondrement actuel de cette activité.

- Au Brésil, la question des forêts n'a pas constitué, par le passé, une problématique sectorielle donnant lieu à une véritable " politique forestière »; elle a plutôt pris la forme d'une problématique foncière dominée par la question des territoires indigènes et de l'accès à la terre, sans compter l'expression d'un nationalisme affirmé face aux tentatives ressenties d'internationalisation de l'Amazonie à travers la rhétorique des biens publics mondiaux (dont certains voudraient que les forêts tropicales fassent partie). La création de concessions d'exploitation forestière sur les forêts publiques d'Amazonie peut être vue comme l'émergence $d$ 'une politique sectorielle pouvant conduire à une représentation modifiée de la forêt autour du problème de sa gestion en tant que ressource boisée.

- En Afrique, essentiellement dans le bassin du Congo et dans certains pays d'Afrique de l'Ouest, la question forestière reste sectorielle et dominée par le rôle de l'exploitation avec ses corollaires : la gestion durable des concessions, la fiscalité (comment capter la rente 
économique) et l'industrie du bois. Depuis quelques années, la contestation de ce régime sectoriel se précise à travers le thème de la foresterie communautaire et, tout récemment, par l'émergence de la problématique de l'autochtonie en République démocratique du Congo : la plainte, largement couronnée de succès, d'associations pygmées contre la Banque mondiale auprès du panel d'inspection de cet organisme a été puissamment relayée par des grandes ONG internationales et la question des "peuples indigènes ", presque ignorée en Afrique jusqu'alors, devient un nouveau vecteur de contestation de l'exploitation industrielle.

\section{Le rôle des ONG}

Les organisations non gouvernementales prennent une importance croissante dans le débat forestier international et contribuent sans aucun doute à façonner les contours disparates d'un régime émergent. Leur influence s'exerce souvent plus par le truchement du lobbying et des pressions auprès des grands organismes de coopération et de développement, comme la Banque mondiale, que par leur influence directe sur les États du Sud. Mais, dans les pays d'Afrique comme dans plusieurs autres pays tropicaux, le front des ONG n'est pas homogène : on a, d'un côté des courants qui veulent favoriser la foresterie communautaire et les droits des peuples «autochtones » et, de l'autre, les ONG de conservation, qui souhaitent une préservation stricte des forêts. Cette préservation est, en effet, peu compatible dans les faits avec la foresterie communautaire, car cette dernière implique souvent le développement du secteur, très actif et peu régulé, de l'exploitation artisanale du bois.

\section{Le mécanisme de réduction des émissions issues de la déforestation et de la dégradation}

La lutte contre les changements climatiques a pour conséquence la promotion de la question des forêts tropicales à un rang très élevé dans l'agenda des négociations internationales sur l'environnement. On peut se demander si, de la même manière qu'un régime de lutte contre les changements climatiques se constitue autour d'accords internationaux (Protocole de Kyoto et autres accords de la Convention-cadre des Nations unies sur les changements climatiques) et autour d'instruments de marché (marché international des permis d'émissions comprenant le mécanisme de développement propre), un régime international des forêts ne va pas se constituer comme un sous-ensemble du régime de lutte contre les changements climatiques. L'éventualité de la mise en place d'un mécanisme international de réduction des émissions issues de la déforestation et de la dégradation (REDD), visant à rémunérer la « déforestation et la dégradation évitées » (dispositif mentionné dans la déclaration finale de la conférence de Bali qui s'est tenue quelques jours après l'atelier), donne du crédit à une telle perspective.

Il y a débat sur la capacité d'un tel mécanisme à enrayer la déforestation, tant la complexité des facteurs qui sous-tendent les rythmes de déforestation est patente, et tant politiques et paramètres extrasectoriels (taux d'intérêts, parités monétaires, politiques agricoles, prix relatifs, demande mondiale d'agrocarburants et de viande...) ont de nombreux effets incertains sur l'évolution du couvert forestier et très dépendants du contexte. La manière dont les États vont réagir à des incitations et des pressions contradictoires (incitations financières à moyen terme à réduire la déforestation, mais coûts politiques et sociaux immédiats à combattre les pratiques conduisant à la déforestation) reste une question ouverte, qui relève plus de l'économie politique que de la science du climat. Les «questions méthodologiques » à résoudre, comme le choix et la construction du scénario de référence (calé sur la déforestation passée ou sur une prédiction de la déforestation future?), la manière de prendre en compte la «dégradation », n'impliquent pas tant des solutions techniques que des choix et des arbitrages politiques, dans la mesure où les règles adoptées engendreront des gagnants et des perdants dans ce qui apparaît devoir constituer une nouvelle rente dont aucun grand pays forestier n'entend être exclu.

Le risque d'un effet en retour sur le marché des permis d'émission a été évoqué. On peut craindre, en effet, un déferlement massif de crédits carbone issus de REDD sur un marché du carbone qui a déjà bien du mal à tenir suffisamment élevé le «signal-prix » de la tonne de $\mathrm{CO}_{2}$ évitée. La réponse apportée par plusieurs participants est qu'un durcissement des objectifs de réduction des émissions dans les pays industrialisés permettrait $d$ 'absorber ces nouveaux crédits. Mais d'autres expriment des doutes quant à cet ajustement possible qui n'est qu'un pari sur les engagements futurs et n'aura rien d'automatique. D'autant qu'une bonne partie des crédits issus de ce mécanisme pourrait bien être non additionnelle (c'est-à-dire découlant simplement de scénarios de référence inappropriés ou manipulés) et constituer de «l'air chaud » (hot air) dont l'effet sur le marché du carbone serait comparable à $l^{\prime}$ injection en grande quantité de fausse monnaie dans un circuit monétaire. D'où les propositions visant à maintenir le mécanisme REDD hors du marché du carbone issu de Kyoto, en versant aux pays de l'argent plutôt que des crédits carbone, afin d'éviter de faire chuter le cours de ces derniers.

Certaines architectures et conséquences possibles du mécanisme REDD suscitent des critiques de la part des ONG qui défendent les populations locales et les peuples 
autochtones ; elles craignent une mainmise étatique accrue sur les forêts aux dépens de la foresterie communautaire, une conservation durcie se traduisant par l'exclusion des populations de l'enceinte des forêts, érigées en forteresses et en réservoirs de carbone rémunérateurs. La question du coût de réduction de la déforestation à travers le calcul du coût d'opportunité du renoncement au déboisement (par exemple, le montant des compensations financières à verser à un paysan pour qu'il renonce à étendre ses champs en défrichant des forêts) a fait l'objet de débats. Un tel calcul est-il pertinent? En effet, le coût réel d'une réduction durable du déboisement est sans doute bien supérieur à la valeur annuelle de la production à laquelle un agent économique doit renoncer, dans la mesure où la mise en œuvre d'un programme capable d'intégrer un grand nombre de ces agents, de leur fournir des alternatives productives et de s'assurer du respect des contrats n'est pas sans impliquer d'autres coûts, que les économistes nomment « coûts de transaction ». Sans oublier quelques problèmes éthiques potentiels, comme le fait de placer les paysans dans une perspective d'assistanat plus ou moins longue, plutôt que dans une position d'acteurs de leur propre développement.

Néanmoins, le mécanisme REDD pourrait jouer un rôle-clé dans le régime international de lutte contre les changements climatiques, dans la mesure où il représenterait l'un des premiers pas vers l'intégration des pays en développement dans le processus d'adoption d'objectifs quantifiés de réduction de leurs émissions futures; cela conforterait la démarche entamée avec le Protocole de Kyoto et remise en cause notamment par l'administration Bush, qui n’a jamais ratifié celui-ci.

L'architecture et les règles du mécanisme REDD sont encore ouvertes et vont faire l'objet de débats entre scientifiques, de négociations techniques et politiques; il pourra en résulter divers régimes de lutte contre la déforestation, aux contours très différents. Ainsi, la rémunération inconditionnelle des gouvernements, avec des crédits carbone "Kyoto", au regard d'un niveau de référence de déforestation (passé ou anticipé) n'est pas la seule option possible. L'idée d'un fonds mondial contre la déforestation a été évoquée. Un tel fonds permettrait de financer des politiques et des mesures dont tout le monde s'accorde pour dire qu'elles sont nécessaires et potentiellement efficaces pour réduire le déboisement, comme la modification des pratiques agricoles et une intensification écologique de l'agriculture, la réforme $\mathrm{du}$ fonctionnement des institutions de contrôle (lutte contre la corruption) et l'évolution des systèmes fonciers en zone forestière. L'autre avantage d'un fonds serait de pouvoir rémunérer les acteurs locaux, et non pas les seuls gouvernements, à travers des programmes de paiements pour services environnementaux à grande échelle, à destination des paysans, des entreprises et des communautés locales.

Mais le financement d'un tel fonds pose le problème, récurrent avec ce type d'instruments, d'un financement durable et à la hauteur des besoins. Si la Norvège a annoncé, peu avant la conférence de Bali, qu'elle consacrerait environ 500 millions de dollars chaque année pendant cinq ans à la lutte contre la déforestation, rien ne dit que beaucoup d'autres pays ont la volonté et les moyens de lui emboîter le pas. Seule la perspective d'une taxe internationale destinée à alimenter ce fonds pourrait changer la donne. La proposition du ministre français de l'Écologie, de l'Énergie, du Développement durable et de l'Aménagement du territoire, Jean-Louis Borloo, en faveur d'une taxation des transactions financières internationales afin de dégager des ressources pour la lutte contre les changements climatiques, trace de telles perspectives, mais une telle mesure n'a pas encore été, elle non plus, mise en place.

Enfin, changer les modèles de consommation, collectifs et individuels, est indispensable : les forêts sont converties en champs de soja (afin de nourrir le bétail face à une consommation croissante de bœuf) ou en cultures de palmiers à huile (stimulées par la demande d'agrocarburants); l'augmentation de la consommation de papier conduit à déboiser les forêts dégradées d'Indonésie pour planter des essences à croissance rapide... Les labels et les instruments économiques ne peuvent, à eux seuls, venir à la rescousse des forêts tropicales. 